\title{
Encoding Organizational Source and Associative Source under Incidental and Intentional Learning Conditions
}

\author{
Lei Zhu \\ Department of Psychology, Fudan University, Shanghai, China \\ Email: judy198181@hotmail.com
}

Received May $24^{\text {th }}, 2013$; revised July $15^{\text {th }}, 2013$; accepted August $6^{\text {th }}, 2013$

\begin{abstract}
Copyright (C) 2013 Lei Zhu. This is an open access article distributed under the Creative Commons Attribution License, which permits unrestricted use, distribution, and reproduction in any medium, provided the original work is properly cited.
\end{abstract}

\begin{abstract}
The present study aimed at providing a plausible answer for the controversy whether organizational source or associative source could be encoded in a more automatic manner through incidental learning. In the experiment, subjects were asked to learn organizational and associative sources under incidental or intentional learning conditions. It turned out that only associative source accuracy increased when subjects were instructed to intentionally learn that source, which implied that associative source might be encoded in a more effortful way, whereas processing of organizational source might be performed incidentally.
\end{abstract}

Keywords: Organizational Source; Associative Source; Incidental Learning; Intentional Learning

\section{Introduction}

People encounter lots of information from various sources every day. Sometimes, when they retrieve the remembered information, they are also required to identify the related source of this information. In the typical source memory tasks, subjects were presented with items from at least two different sources (e.g., Zhu et al., 2010). The items to be learned include words, pictures, statements, and so on. And the source refers to a variety of characteristics which specify the conditions under which the learning items are presented, such as their color, shape, size, spatial location, temporal order and the media or modality of presentation.

In the recent decades, there is increasing evidence which suggested that source memory might be fundamentally different from memory for the item. That is, compared to memory formation for item, encoding source is a more effortful task which is performed intentionally and requires considerable attentional resources (Spencer \& Raz, 1995; Troyer \& Craik, 2000). For example, the population with decline of frontal executive systems and reduced attentional resources, such as older adults and amnesia patients showed more deficits in remembering the sources than in remembering the items (Schacter, 1987; Schacter et al., 1991; Ferguson et al., 1992; Hashtroudi et al., 1994; Chalfonte et al., 1996; Wegesin et al., 2000). In addition, encoding source was interfered more severely by the secondary tasks under divided attention condition than was encoding item (Troyer et al., 1999; Troyer \& Craik, 2000). Similarly, compared with intentional learning conditions, source accuracy decreased under incidental learning conditions (Chalfonte \& Johnson, 1996; Marsh et al., 2004; Kuo \& Van Petten, 2006; Uncapher \& Rugg, 2009).

However, not all the source is encoded in the same way. One insight comes from the dichotomy between automatic and effortful processes proposed by Hasher and Zacks (1979). Ac- cording to Hasher and Zacks (1979), automatic memory processes which require minimal attentional resources can occur without intention and do not benefit from practice. Contrarily, effortful memory processes such as rehearsal or elaborative mnemonic activities which require considerable attentional resources are initiated intentionally and show benefits from practice. Based on that, Hasher and Zacks (1979) further proposed that different from other source, spatial locations, temporal orders tend to be encoded incidentally by automatic memory processes. Yet, on the contrary, other researchers classified source into two categories (Geiselman \& Bjork, 1980; Baddeley, 1982; Moscovitch, 1992; Spencer \& Raz, 1995; Staresina \& Davachi, 2008). Opposite to Hasher and Zacks (1979), source, such as spatial locations or temporal orders, is thought to be extrinsic to the item and not be encoded concurrently with the item. Thus, more intentional effort is required to bind it to the item. This type of source has been named as extrinsic (Geiselman \& Bjork, 1980; Baddeley, 1982), organizational (Moscovitch, 1992), spatiotemporal (Spencer \& Raz, 1995), or item-context (Staresina \& Davachi, 2008) source. On the other hand, source, such as color, font, shape, is likely to be encoded automatically without conscious intention, because it is directly bound to the item itself and is encoded concurrently with the item. This type of source is denominated as intrinsic (Geiselman \& Bjork, 1980; Baddeley, 1982), associative (Moscovitch, 1992), stimulus bound (Spencer \& Raz, 1995), or itemfeature (Staresina \& Davachi, 2008) source. Thus, it is still an open question whether organizational source or associative source could be encoded in a more automatic manner through incidental learning.

To test this, participants were asked to encode organizational source (i.e., location) and associative source (i.e., color) under incidental or intentional learning conditions. They first studied a list of colored objects presented in the top or bottom of the 
computer screen during three tasks (Figure 1). In the itemoriented task, participants judged whether the items depicted in pictures were animate. In the color-oriented task, they judged whether the item-color conjunctions were plausible (red hat) or not (green orange) in real life. In the location-oriented task, they judged whether it was plausible in the real life that the item appeared in the sky when the item was presented in the top of the screen and judged whether it was plausible in the real life that the item appeared in the ground when the item was presented in the bottom of the screen. Thus, organizational source was intentionally learned in location-oriented task, but was incidentally learned in the other two tasks, whereas associative source was intentionally learned in color-oriented task, but was incidentally learned in the other two tasks. It should be noted that such manipulation of intentional and incidental learning is different from the traditional Craik and Lockhart (1972) definition for item memory. The Craik and Lockhart (1972) distinguish intentional and incidental learning by explicit instruction to learn specific item, whereas the present study distinguish intentional and incidental learning by the encoding task. After that, participants took a surprised subsequent memory test where they first gave old/new decision, and then, for items called old, they gave source (color and location) judgment. It is predicted that if the associative source needs to be encoded intentionally by effortful memory processes, then the source accuracy of color would benefit from intentional learning. On the other hand, if organizational source is encoded through intentional learning, the source accuracy of location would differ for three tasks.

\section{Method}

\section{Participants}

Fourteen right-handed Chinese volunteers from the university community with normal or corrected-to-normal vision (three

(a) Encoding

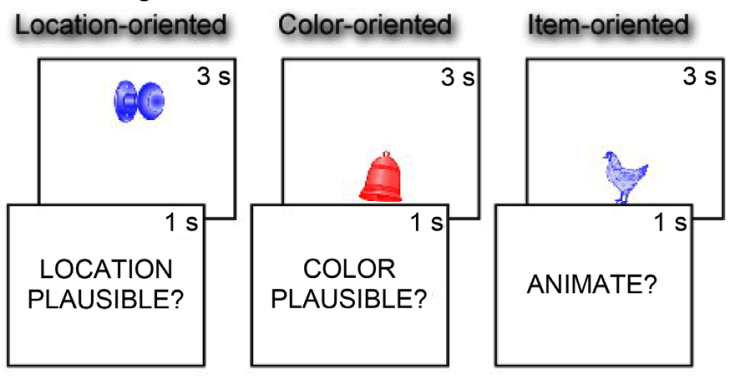

(b) Test

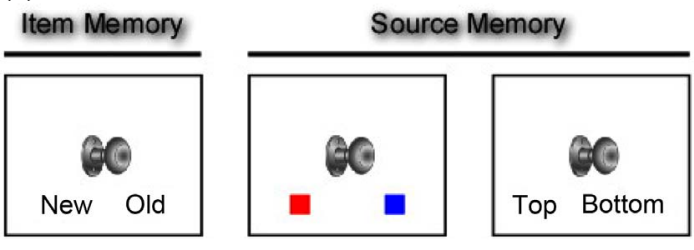

Figure 1.

Experimental Design. (a) Encoding phase. The task cues were varied between trials and participants were instructed to conduct location-oriented task, color-oriented task or item-oriented task according to the task cue; (b) Subsequent memory test. For the items judged as "old" in the item memory test, their source memory for location and color was further assessed. males, aged from 19 to $29, M=22.00, S D=3.11$ ) participated in this experiment. All the participants were naïve about the purpose of the experiment and were paid $20 \mathrm{RMB}$ for their participation.

\section{Materials}

Two hundred and forty gray pictures of common objects were randomly selected from the revised Snodgrass and Vanderwart's object pictorial set (Rossion \& Pourtois, 2004) as materials, 180 of which were randomly selected to serve as learning items with 60 for each task condition and 60 of which served as lures during the memory test. The learning items in each condition were randomly transferred into two different colors (red [RGB: 255, 0, 0] or blue [RGB: 0, 0, 255]), with 30 items in each color. Then, 30 items in each color for each task were randomly assigned to two locations, with 15 items in each location. Complexity, agreement, imagery and familiarity of the items were counterbalanced across different conditions, different colors and different locations. The pictures subtended about $10^{\circ}$ vertical and horizontal visual angles in the encoding phase and $12^{\circ}$ in the test phase at the viewing distance of about $50 \mathrm{~cm}$. All the stimuli were presented on a white screen with a resolution of $640 \times 480$ pixels.

\section{Procedures}

The participants were not informed about the subsequent memory test before the task. During the encoding phase, 180 learning items were presented sequentially (Figure 1). For each encoding trial, a $1 \mathrm{~s}$ task cue was presented at first, followed by a $3 \mathrm{~s}$ colored item. The task cues were varied between trials and participants were required to judge whether it was plausible to encounter the item appearing in the sky (when the item appear in the top) or ground (when the item appear in the bottom) when viewing "LOCATION PLAUSIBLE?" (location-oriented task), but judge whether it was plausible to encounter the item appearing in the given color in real life when viewing "COLOR PLAUSIBLE?" (color-oriented task). They were also asked to judge whether the item depicted in the picture was animate when viewing "ANIMATE?" (item-oriented task). Reponses were given via participant's key press. The order of presentation sequence was randomized across participants.

About five minutes after the encoding phase, participants were given a surprised self-paced memory test. 240 gray objects were presented sequentially in a random order across participants. Participants were instructed to indicate whether the object was old (presented during the encoding phase) or new (not presented during the encoding phase). If they responded as "old", they were then required to indicate the related location and color of the object. The order of the location and color judgments were counterbalanced between participants.

\section{Results}

\section{Encoding Task}

The encoding trials with no responses $(0.16 \% \pm 0.34 \%$ of trials) were excluded from further analyses. As is shown in Table $\mathbf{1}$, the location-oriented trials and the color-oriented trials produced similar rates of "YES" responses (judged to be plausible and animate for all the trials, $t(13)=0.49, p>.05,1-\beta=0.07$ ), whereas the proportions of "YES" responses in both tasks were 
Table 1.

Behavioral performance during encoding and subsequent memory test.

\begin{tabular}{cccc}
\hline Task & $\begin{array}{c}\text { "YES" proportion } \\
(\%)\end{array}$ & $\begin{array}{c}\text { Encoding RT } \\
(\mathrm{ms})\end{array}$ & $\begin{array}{c}\text { Recognition } \\
\text { (hit rate \%) }\end{array}$ \\
\hline Location-oriented & $56.79(9.57)$ & $1330(190)$ & $73.33(14.75)$ \\
Color-oriented & $54.34(16.18)$ & $1431(217)$ & $78.94(10.34)$ \\
Item-oriented & $32.29(6.04)$ & $1072(184)$ & $67.69(14.48)$ \\
\hline
\end{tabular}

Note: Standard errors are given in parentheses. Average "yes" proportions and recognition hit rates (\%) across participants is shown as a proportion of all valid encoding trials within each task.

higher than that in the item-oriented task ( $t \mathrm{~s}>4.71, p \mathrm{~s}<.05$ with Bonferroni correction for multiple comparison). Besides, participants responded faster in the item-oriented task than in the location-oriented task $(t(13)=9.50, p<.05$ with Bonferroni correction for multiple comparison) and responded faster in the location-oriented task than in the color-oriented task $(t(13)=$ $2.46, p<.05$ with Bonferroni correction for multiple comparison).

\section{Memory Test}

Turning to the subsequent memory performance during test, the learning items were recognized at a rate of $73.32 \% \pm$ $11.63 \%$ and lures were correctly rejected at a rate of $86.31 \% \pm$ 7.34\%. As shown in Table 1, the mean recognition hit rate in the color-oriented task was significantly higher than that in the item-oriented task $(t(13)=3.87, p<.05$ with Bonferroni correction for multiple comparison). The difference of hit rates between the color-oriented and the location-oriented, the location-oriented and the item-oriented conditions did not reach statistical significance.

Last, the source accuracy of location and color in three tasks, taken as the proportion of correctly recognized trials, are illustrated in Figure 2. A 3 task * 2 source ANOVA revealed that the main effect of source $(F(1,13)=60.12, p<.01)$, the main effect of task $(F(2,26)=14.54, p<.01)$ and the interaction $(F(2,26)=18.64, p<.01)$ were significant. This result indicated that the source accuracy of location was higher than that of color. Further analysis revealed the source accuracy in the color-oriented task was higher than in the other two tasks ( $t \mathrm{~s}>$ $4.14, p s<.05$ with Bonferroni correction for multiple comparison), whereas the difference of source accuracy between the the location-oriented and the item-oriented tasks did not reach statistical significance $(t(13)=1.44, p>.05,1-\beta=0.27)$. Besides, simple effect analysis of the task effect revealed that the effect did not differ from zero for location judgment $(F(2,26)=$ $1.67, p>.05,1-\beta=0.32)$, but was significantly greater than zero for color judgment $(F(2,26)=30.35, p<.01)$. Further analysis revealed that the source accuracy of color in the color-oriented task was higher than that in the other two tasks (ts $>5.98, p \mathrm{~s}<.05$ with Bonferroni correction for multiple comparison), whereas the difference of source accuracy of color between the location-oriented and the item-oriented tasks did not reach statistical significance $(t(13)=0.41, p>.05,1-\beta$ $=0.07)$. These findings suggested that the associative source (i.e., color) needs to be encoded intentionally because its source accuracy benefited from intentional learning, whereas the organizational source (i.e., location) could be encoded in a more automatic manner.

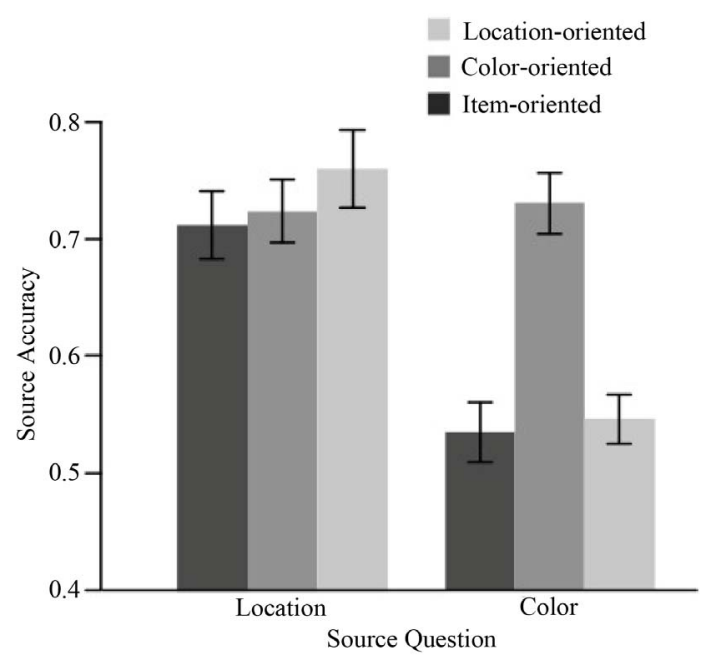

Figure 2.

Average source accuracy of location and color in three tasks. Eorror bars indicates $+/-$ standard errors.

\section{Discussion}

The present study aimed at providing a plausible answer for the controversy whether organizational source or associative source could be encoded in a more automatic manner through incidental learning. Consistent with Hasher and Zacks (1979), the results implied that organizational source might be encoded in a more automatic manner through incidental learning, but associative source might be encoded in a more effortful way, since only associative source accuracy increased when subjects were instructed to intentionally learn that source. However, as Hasher and Zacks's (1979) viewpoint, automatic and effortful memory processes can be distinguished from three aspects: whether it requires considerable attentional resources; whether it can occur without intention; and whether it benefits from practice. The present study tested whether the processes involved in encoding organizational or associative source could occur without intention. Whether encoding organizational or associative source requires considerable attentional resources or whether it benefits from practice remains to be tested.

The findings that relative to associative source, organizational source might be encoded in a more automatic manner through incidental learning does not comply with the predictions by many other researchers, who argued that organizational source should be encoded in a more effortful manner, since it is extrinsic to the item and more intentional effort is required to bind it to the item (Geiselman \& Bjork, 1980; Baddeley, 1982; Moscovitch, 1992; Spencer \& Raz, 1995; Staresina \& Davachi, 2008). This inconsistency might be due to the superiority of learning and memory associated with location. Location changes may involve eye movements, which may facilitate learning and memory (Mayr, 1996) and counteract the interruption of main task in the present study. Further investigation could focus on the dissociation between associative sources and other organizational sources, such as time.

Besides, the results of the present study could be used to explain the results from previous studies using incidental learning task. Previous studies demonstrated that organizational source accuracy was higher than associative source accuracy during incidental learning (i.e., participants were not informed what 
would be tested later and the related source was not a part of the demand of encoding task) (Staresina \& Davachi, 2008, Uncapher et al., 2006). Based on the present results, it is suggested that organizational source is encoded concurrently with the main task, which produces significantly higher performance than associative source. Consistently, our data also showed that organizational source accuracy was higher than associative source accuracy in the item-oriented task $(\mathrm{t}(13)=6.07, p<.05$ with Bonferroni correction for multiple comparison).

It should be noted that the results of the present study could not merely be explained by congruency effect. According to congruency effect, congruous items eliciting "yes" answers (e.g., "is ELEPANT animate?") will result in better subsequent memory than incongruous items eliciting "no" answers (e.g., "is TABLE animate?", e.g., Schulman, 1974; Craik \& Tulving, 1975; Staresina et al., 2009), because unlike incongruous items, the congruous items have a preexisting relationship with the question context. This relationship is thought to prompt additional relational binding processes to combine the context and the item as an integrated unit, which in turn strengthens the memory trace. The present study found the location-oriented task and the color-oriented task produced more "YES" responses than the item-oriented task, which might foster additional relational binding processes and strengthen the memory trace. However, although the source accuracy in the color-oriented task was higher than in the item-oriented task, such superiority in source memory did not appear for the location-oriented task.

Finally, it should also be noted that the results of the present study might not be strategy-derived. The present experiment used a mixed trials approach at encoding phase (i.e., asking participants to make different types of judgments from trial-totrial), which might lead participants to more intentionally encode all aspects of the stimulus in each trial and develop strategies relating to the perceived importance of location, color, and animacy. Correspondingly, one might argue that the present results might be strategy-derived, rather than truly representing the difference between incidental and intentional encodings. However, our experimental methods rule out such possibility. In the experiment, the frequency of each task $(1 / 3)$ remains the same during encoding phase. Such manipulation, which has been informed to the participants before the experiment, might result in equal perceived importance of three aspects (location, color, and animacy).

In sum, the present findings suggested that associative source might be encoded in more effortful way, whereas processing of organizational source might be performed incidentally.

\section{Acknowledgements}

This research was supported by National Natural Science Foundation of China (31100728), Projects Planning in Shanghai Philosophy and Social Sciences Research (2012JJY001).

\section{REFERENCES}

Baddeley, A. D. (1982). Domains of recollection. Psychological Review, 89, 708-729. doi:10.1037/0033-295X.89.6.708

Chalfonte, B. L., \& Johnson, M. K. (1996). Feature memory and binding in young and older adults. Memory \& Cognition, 24, 403-416. doi:10.3758/BF03200930

Chalfonte, B. L., Verfaellie, M., Johnson, M. K., \& Reiss, L. (1996). Spatial location memory in amnesia: binding item and location in- formation under incidental and intentional encoding conditions. $\mathrm{Me}$ mory, 4, 591-614. doi:10.1080/741940998

Craik, F. I. M., \& Tulving, E. (1975). Depth of processing and the retention of words in episodic memory. Journal of Experimental Psychology: General, 104, 268-294. doi:10.1037/0096-3445.104.3.268

Ferguson, S. A., Hashtroudi, S., \& Johnson, M. K. (1992). Age differences in using source-relevant cues. Psychology and Aging, 7, 443452. doi: $10.1037 / 0882-7974.7 .3 .443$

Geiselman, R. E., \& Bjork, R. A. (1980). Primary versus secondary rehearsal in imagined voices: Differential effects on recognition. Cognitive Psychology, 12, 188-205. doi:10.1016/0010-0285(80)90008-0

Hashtroudi, S., Johnson, M. K., Vnek, N., \& Ferguson, S. A. (1994) Aging and the effects of affective and factual focus on source monitoring and recall. Psychology and Aging, 9, 160-170. doi:10.1037/0882-7974.9.1.160

Kuo, T. Y., \& Van Petten, C. (2006). Prefrontal engagement during source memory retrieval depends on the prior encoding task. Journal of Cognitive Neuroscience, 18, 1133-1146. doi:10.1162/jocn.2006.18.7.1133

Marsh, R. L., Hicks, J. L., \& Cook, G. I. (2004). Focused attention on one contextual attribute does not reduce source memory for a different attribute. Memory, 2004, 12, 183-192. doi: $10.1080 / 09658210344000008$

Mayr, U. (1996). Spatial attention and implicit sequence learning: Evidence for independent learning of spatial and non spatial sequences. Journal of Experimental Psychology: Learning, Memory and Cognition, 22, 350-364. doi:10.1037/0278-7393.22.2.350

Moscovitch, M. (1992). Memory and working-with-memory: A component process model based on modules and central systems. Journal of Cognitive Neuroscience, 4, 257-267. doi:10.1162/jocn.1992.4.3.257

Rossion, B., \& Pourtois, G. (2004). Revisiting Snodgrass and Vanderwart's object set: The role of surface detail in basic-level object recognition. Perception, 33, 217-236. doi:10.1068/p5117

Schacter, D. L. (1987). Memory, amnesia, and frontal lobe dysfunction. Psychobiology, 15, 21-36.

Schacter, D. L., Kaszniak, A. W., Kihlstrom, J. F., \& Valdiserri, M. (1991). The relation between source memory and aging. Psychology and Aging, 6, 559-568. doi:10.1037/0882-7974.6.4.559

Schulman, A. I. (1974). Memory for words recently classified. Memory \& Cognition, 2, 47-52. doi:10.3758/BF03197491

Spencer, W. D., \& Raz, N. (1995). Differential effects of aging on memory for content and context: A meta-analysis. Psychology and Aging, 10, 527-539. doi:10.1037/0882-7974.10.4.527

Staresina, B. P., \& Davachi, L. (2008). Selective and shared contributions of the hippocampus and perirhinal cortex to episodic item and associative encoding. Journal of Cognitive Neuroscience, 20, 1478 1489. doi:10.1162/jocn.2008.20104

Staresina, B. P., Gray, J. C., \& Davachi, L. (2009). Event congruency enhances episodic memory encoding through semantic elaboration and relational binding. Cerebral Cortex, 19, 1198-1207. doi:10.1093/cercor/bhn165

Troyer, A. K. \& Craik, F. I. (2000). The effect of divided attention on memory for items and their context. Canadian Journal of Experimental Psychology, 54, 161-171. doi:10.1037/h0087338

Troyer, A. K., Craik, F. I. M., Winocur, G., \& Moscovitch, M. (1999). Source memory and divided attention: Reciprocal costs to primary and secondary tasks. Neuropsychology, 13, 467-474. doi:10.1037/0894-4105.13.4.467

Uncapher, M. R., \& Rugg, M. R. (2009) Selecting for Memory? The influence of selective attention on the mnemonic binding of contextual information. The Journal of Neuroscience, 29, 8270-8279. doi:10.1523/JNEUROSCI.1043-09.2009

Uncapher, M. R., Otten, L. J., \& Rugg, M. D. (2006). Episodic encoding is more than the sum of its parts: An fMRI investigation of multifeatural contextual encoding. Neuron, 52, 547-556. doi:10.1016/j.neuron.2006.08.011

Wegesin, D. J., Jacobs, D. M., Zubin, N. R., Ventura1, P. R., \& Stern, Y. (2000). Source memory and encoding strategy in normal aging. 
Journal of Clinical and Experimental Neuropsychology, 22, 455-464. doi:10.1076/1380-3395(200008)22:4;1-0;FT455

Zhu, L., Guo, X., Zheng, L., Li, J., Pei, M., Dienes, Z., \& Yang, Z.
(2010). Graded contribution of hippocampus to multifeature binding across temporal delay. Neuroreport, 21, 902-906. doi:10.1097/WNR.0b013e32833dddb4 